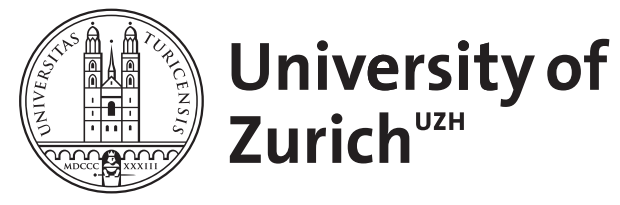

\title{
Vergilrezeption im deutschen Humanismus am Beispiel von Stephan Reichs 'Bucolica'-Übersetzung
}

\author{
Frick, Julia
}

DOI: https://doi.org/10.1515/9783110527230-010

Posted at the Zurich Open Repository and Archive, University of Zurich ZORA URL: https://doi.org/10.5167/uzh-147636

Book Section

Published Version

Originally published at:

Frick, Julia (2017). Vergilrezeption im deutschen Humanismus am Beispiel von Stephan Reichs 'Bucolica'Übersetzung. In: Toepfer, Regina; Kipf, Johannes Klaus; Robert, Jörg. Humanistische Antikenübersetzung und frühneuzeitliche Poetik in Deutschland (1450-1620). Berlin, Boston: De Gruyter, 177-194. DOI: https://doi.org/10.1515/9783110527230-010 
Humanistische Antikenübersetzung und frühneuzeitliche Poetik in Deutschland (1450-1620) 


\section{Frühe Neuzeit}

Studien und Dokumente zur deutschen Literatur und Kultur im europäischen Kontext

Herausgegeben von

Achim Aurnhammer, Wilhelm Kühlmann, Jan-Dirk Müller, Martin Mulsow und Friedrich Vollhardt

Band 211 


\section{Humanistische Antikenübersetzung und frühneuzeitliche Poetik in Deutschland (1450-1620)}

Herausgegeben von

Regina Toepfer, Johannes Klaus Kipf und Jörg Robert 
ISBN 978-3-11-052606-6

e-ISBN (PDF) 978-3-11-052723-0

e-ISBN (EPUB) 978-3-11-052634-9

ISSN 0934-5531

\section{Library of Congress Cataloging-in-Publication Data}

A CIP catalog record for this book has been applied for at the Library of Congress.

\section{Bibliografische Information der Deutschen Nationalbibliothek}

Die Deutsche Nationalbibliothek verzeichnet diese Publikation in der Deutschen Nationalbibliografie; detaillierte bibliografische Daten sind im Internet über http://dnb.dnb.de abrufbar.

(C) 2017 Walter de Gruyter GmbH, Berlin/Boston

Druck und Bindung: $\mathrm{CPI}$ books $\mathrm{GmbH}$, Leck

@ Gedruckt auf säurefreiem Papier

Printed in Germany

www.degruyter.com 


\section{Inhalt}

Regina Toepfer, Johannes Klaus Kipf, Jörg Robert

Einleitung: Humanistische Antikenübersetzung und frühneuzeitliche Poetik in Deutschland (1450-1620) - 1

\section{Sektion I: Übersetzungsreflexion und Sprachbewusstsein}

Regina Toepfer

Einleitung: Übersetzungsreflexion und Sprachbewusstsein -27

Jan-Dirk Müller

Parameter des Übersetzens - 33

Klaus Grubmüller

Widererwaxsung

Anmerkungen zur sprachgeschichtlichen Bedeutung des deutschen

Humanismus -57

Thomas Baier

Erasmus als Übersetzer — 73

Lydia Wegener

wie das ein grosser vnderscheyd seye zwischen disen Teütschen vnd den vorigen Lateinischen Josephis

Zur Umsetzung von Caspar Hedios Überbietungsanspruch im Josephus

Teütsch - 93

\section{Sektion II: Institutionen und Funktionen}

Regina Toepfer

Einleitung: Institutionen und Funktionen -119

Christa Bertelsmeier-Kierst

Übersetzen im deutschen Frühhumanismus

Ergebnisse des MRFH zur Einbürgerung humanistischer und antiker Autoren bis $1500-125$ 
Carola Redzich

Vergil zü tütsch

Zur Programmatik der ,Klassiker'-Übersetzung in Adelphus Mulings Hirten

büch (1508/12) und Thomas Murners Aeneadischen Büchern (1515) —-151

Julia Frick

Vergilrezeption im deutschen Humanismus am Beispiel von Stephan Reichs Bucolica-Übersetzung — 177

Christoph Galle

Lateinische Texte der Humanisten Hutten, Reuchlin, Erasmus und ihre volkssprachigen Übersetzungen in der Reformationszeit

Mit einem Verzeichnis der Schriften und Übersetzungen Huttens

1517-1530-195

\section{Sektion III: Intermedialität und Paratextualität}

Jörg Robert

Einleitung: Intermedialität und Paratextualität - 217

Seraina Plotke

Humanistische Transpositionen

Die ersten deutschen Übersetzungen von Andrea Alciatos

Emblembuch - 223

Marion Gindhart

Bildschrift im Kontext

Die Hieroglyphica-Übersetzung Johannes Herolds (Basel 1554) — 243

Manfred Kern

Metáphrasis und Metaphorá

Über emblematische Verfahren in den deutschen Übersetzungen antiker

Großepik (Minervius' Odyssea und Wickrams Metamorphosen) — 287 


\section{Sektion IV: Poetik und Rhetorik}

Jörg Robert

Einleitung: Poetik und Rhetorik — 315

Joachim Hamm

Antikenübersetzung, frühneuzeitliche Poetik und deutscher Prosastil

Zur Bamberger Übertragung von Ciceros Cato maior de senectute

(1522) -323

Jörg Robert

Luthers Lieder als Antikenübersetzung?

Überlegungen zur Ambrosius-Bearbeitung Nu kom der Heyden

heyland -353

Regina Toepfer

Veranschaulichungspoetik in der frühneuhochdeutschen Ovid-Rezeption

Philomelas Metamorphosen bei Wickram, Spreng und Posthius _ 383

Jörg Wesche

Trügerische Antikenübersetzung

Poetologisches Translationsverständnis bei Martin Opitz und humanistische

Autorisierung im Lob des Feldtlebens (1623) - 409

\section{Sektion V: Literaturvarianten und Gattungstransfer}

Johannes Klaus Kipf

Einleitung: Literaturvarianten und Gattungstransfer -429

Tina Terrahe

Poetologische Transformationen bei Heinrich Steinhöwel - 439

Kerstin Brix

Ein deutscher ,Sueton“

Jakob Vielfelds Übersetzung der Kaiserviten (1536) — 461 
$\mathbf{X}$ - Inhalt

Ralph Häfner

Ciceros Somnium Scipionis in volkssprachigen Übersetzungen des frühen

16. Jahrhunderts (Cammerlander, Janot, Brucioli)

Mit einer Textsynopse im Anhang — 491

Stefan Seeber

Heliodor unter der Tarnkappe

Zschorns Übersetzung der Aithiopika (1559) im Kontext der Zeit — $\mathbf{5 1 1}$

Johannes Klaus Kipf

zugfallen dem gemeinen man, Der sonst doch nicht viel mores kan

Zu den ersten deutschen Übersetzungen von Plautus' Aulularia im

16. Jahrhundert $-\mathbf{5 2 7}$

Ralf Junghanns

Kann kein Latein, hat nie studiert

Zur Vermittlung antiker Stoffe durch Georg Gotthart (gest. 1619) — 557

Register -577 


\section{Vergilrezeption im deutschen Humanismus am Beispiel von Stephan Reichs Bucolica- Übersetzung}

Vergil ist im 16. Jahrhundert mehrfach in deutscher Sprache präsent: In der ersten vollständigen Übersetzung der Bucolica von Adelphus Muling, ${ }^{1}$ der Aeneis-Übersetzung Thomas Murners ${ }^{2}$ sowie in den Bucolica- und Georgica-Translationen Stephan Reichs (Riccius). ${ }^{3}$ Sowohl Muling als auch Murner dokumentieren in den Vorreden ihrer Werke die Wertschätzung Vergils im Zeitalter des Humanismus: Die Bezeichnungen des großen Dichters als des höchberümpten aller latinischen poeten ${ }^{4}$ und des anmütigsten latynschen man vff erden ${ }^{5}$ verweisen auf Vergils zentrale Rolle im lateinischen Bildungsbetrieb der Zeit. Daher gelte es, sein Werk auch denjenigen $\mathrm{zu}$ erschließen, denen aufgrund mangelnder Lateinkenntnisse der Zugang dazu verwehrt sei - Vergil von latynschem todt in tütsches leben zu erquicke[n], wie Murner es in seiner Vorrede unter Rückgriff auf den humanistischen Topos der Wiedererweckung so treffend formuliert. ${ }^{6}$

1 Johann Adelphus Muling: P. Virgilij Bucolica zü tütsch das Hirten vnnd buren werck der .x. Eglogen publy Virgily Maronis von Mantua. Straßburg: Johann Grüninger [1508/09] (VD16 V 1529). Vor Muling fertigte der Heidelberger Humanist Adam Wernher von Themar eine Prosaübersetzung der achten und zehnten Ekloge an, die er im Jahr 1502 dem Pfalzgrafen Philipp widmete, vgl. Franz Josef Worstbrock: Wernher, Adam, von Themar. In: Deutscher Humanismus 1480-1520. Verfasserlexikon 2 (2013), Sp. 1277-1289.

2 Thomas Murner: Vergilij maronis dryzehen Aeneadischen Bücher von Troianischer zerstörung/ vnd vffgang des Römischen Reichs. durch doctor Murner vertütst. Straßburg: Johann Grüninger 1515 (VD16 V 1426).

3 Stephan Reich: Bucolica Virgilii In Usum Puerorum Germanice Reddita Per M. Stephanum Riccium. Leipzig: Hans Rambau d. Ä. für Jakob Apel d. Ä. 1568 (VD16 V 1521). Die Übersetzung der Georgica erschien in zwei Teilen: Stephan Reich: P. Virgilii Maronis Priores Duo Libri Georgicorum In Usum Studiosae Iuventutis Germanice redditi, et editi A M. Stephano Riccio Seniore. Görlitz/Leipzig: Ambrosius Fritsch für Jakob Apel d. Ä. 1571 (VD16 V 1563); Stephan Reich: Posteriores Duo Libri Georgicorum P. Virgilii Maronis in usum Iuventutis Studiosae Germanice redditi, Per M. Stephanum Riccium. Leipzig: Jakob Bärwald für Jakob Apel d. Ä. 1572 (VD16 V 1564).

4 Muling (Anm.1), fol. IIr.

5 Murner (Anm.2), fol. A1v. Die Vorrede ist auch abgedruckt bei Eckhard Bernstein: Die erste deutsche Äneis. Eine Untersuchung von Thomas Murners Äneis-Übersetzung aus dem Jahre 1515. Meisenheim am Glan 1974 (Deutsche Studien 23), S. 105.

6 Murner (Anm. 2), fol. A1v. 
Alle drei Übersetzer verbindet die Intention, ein Hilfsmittel für die Lektüre des lateinischen Originaltextes zu schaffen: Mulings Bucolica-Übersetzung ist den iungen anfahenden schülern zugedacht, damit diese leichtlicher mögent verfassen begreiffen vnd behalten die loblichen schrifften Virgily; ${ }^{7}$ Murner legt den angezielten Rezipientenkreis seiner deutschen Aeneis mit der Gruppe derjenigen, die das römische Recht studieren wollen, fest: zuo wolgefallen allen denen so sich in Vergilio ieben und die nach verfaßtem latyn Keiserliche recht begeren zuo leeren; ${ }^{8}$ Reich spricht vom usus puerorum, ${ }^{9}$ dem Nutzen, den seine Übersetzung der Bucolica für die Studien der jungen Leute bereithalte. Die Wege, die die Übersetzer einschlagen, unterscheiden sich grundlegend voneinander.

Die folgenden Ausführungen sind der Vergilrezeption im deutschen Humanismus am Beispiel von Reichs Bucolica-Übersetzung gewidmet. Dafür wird zunächst kurz Mulings und Murners translatorische Vorgehensweise skizziert (I.), um durch den Vergleich die Eigenart von Reichs Bucolica-Übersetzung herauszuarbeiten (II.); es schließt sich ein Ausblick auf seine Verdeutschung von Vergils Georgica an (III.); den Abschluss bilden Überlegungen zur Art und Weise der Vergillektüre im 16. Jahrhundert (IV.).

\section{Zu Mulings Bucolica- und Murners Aeneis-Übersetzung}

Adelphus Mulings Hirten vnnd buren werck der .x. Eglogen verließ im Jahr 1508/09 die Straßburger Offizin des Johann Grüninger. ${ }^{10}$ Die Übersetzung ist mit den Holzschnitten aus Sebastian Brants großer Gesamtausgabe der Werke Vergils ausgestattet, die Grüninger im Jahr 1502 verlegt hatte. ${ }^{11}$

7 Muling (Anm. 1), fol. IIr.

8 Murner (Anm. 2), fol. A1v.

9 Reich: Bucolica Virgilii (Anm. 3), fol. A1r.

10 Muling (Anm. 1). Franz Josef Worstbrock weist nach, dass die im VD16 angesetzte Datierung auf „[ca. 1520]“ aufgrund der Namensform Mülich und der Tatsache, dass Muling sich nur um 1510 mit der Übersetzung antiker Autoren beschäftigt hat, nicht haltbar ist, vgl. Franz Josef Worstbrock: Adelphus Mulings Vergilübersetzung. In: Zeitschrift für deutsches Altertum 102 (1973), S. 203-210, hier S. 204-206. Zu Mulings Gesamtwerk vgl. Franz Josef Worstbrock: Muling, Johann Adelphus. In: Deutscher Humanismus 1480-1520. Verfasserlexikon 2 (2013), Sp. 255-277. 11 Publii Virgilii Maronis opera cum quinque vulgatis commentariis expolitissimisque figuris atque imaginibus nuper per Sebastianum Brant superadditis exactissimeque revisis atque elimatis. Straßburg: Johann Grüninger 1502 (VD16 V 1332). Zu den Holzschnitten der Ausgabe vgl. Nikolaus Henkel: Das Bild als Wissenssumme. Die Holzschnitte in Sebastian Brants Vergil-Ausgabe, Straßburg 1502. In: Schreiben und Lesen in der Stadt. Literaturbetrieb im spätmittelalterlichen Straßburg. Hg. von Stephen Mossman, Nigel F. Palmer, Felix Heinzer. Berlin 2012 (Kultur- 
Muling will seine Übersetzung als Hilfsmittel zum Erlernen des Lateinischen und zur Lektüre des Originaltextes genutzt wissen - seine Translation ist historisch im Bereich der schulischen Bildung zu verorten. Dem Zweck, den vergilischen Text besser verfassen begreiffen vnd behalten $\mathrm{zu}$ können, dienen Prosa-Argumenta ${ }^{12}$ sowie je vier paarweise gereimte Verse,$^{13}$ die jeder Ekloge vorangehen und deren Inhalt in gebotener Kürze prägnant zusammenfassen. Mulings Intention, den anfahenden schülern die Lektüre des Originals durch dessen Erschließung zu erleichtern, entspricht seine translatorische Vorgehensweise, von der er im Vorwort Rechenschaft ablegt (fol. IIr):

in welchem [sc. dem hohen latyn Virgily] wir vnß geflissen (so vil müglich) das allwege ein yeder verß dem latin mit seiner gloß vnnd vßlegung vergleicht werde/ vnnd eines dem andern correspondiere

Ein Blick auf das Layout der Ausgabe verdeutlicht das dreischrittige Vermittlungsmodell: Der wörtlichen und stichischen Übersetzung eines jeden lateinischen Verses schließt sich eine graphisch untergeordnete Paraphrase an, während die Marginalien die Ebene der Kommentierung bieten. Muling strebt danach, „die Gestalteinheit des vergilischen Verses, in den Grenzen des Deutschen auch die Wortstellung zu bewahren." ${ }^{14}$ Die Heranziehung des lateinischen Originaltextes ist bei dieser Konzeption intendiert.

Muling bereitet den Text entsprechend der Praxis der lateinischen kommentierten Ausgaben in Mittelalter und Früher Neuzeit auf, wo die Glossierung und die Erschließung des Originaltextes durch Interlinearversionen sowie Kommen-

topographie des alemannischen Raums 4), S. 389-419. Zur Nachwirkung der Ausgabe siehe auch Bernd Schneider: Virgilius pictus - Sebastian Brants illustrierte Vergilausgabe von 1502 und ihre Nachwirkung. Ein Beitrag zur Vergilrezeption im deutschen Humanismus. In: Wolfenbütteler Beiträge 6 (1983), S. 202-262.

12 Jeder Ekloge geht ein Argumentum, eine in deutscher Prosa gehaltene Zusammenfassung des Inhalts, voran, wie zum Beispiel das Argumentum zur ersten Ekloge: Melibeus der hürt/gand vnd wichende $v \beta$ den äckeren/felden/vnnd bännen/ dannen die burenn von Mantua vßgeworffen vnnd vertriben wurden mit allem sinem fiehe findet Titirum ein andern hirten ligen vnder dem schatten des buochboms. Muling (Anm.1), fol. IIIr.

13 Mit den deutschen Merkversen werden die spätantiken, zwei Hexameter umfassenden Argumenta wiedergegeben. Vgl. die Verse zur ersten Ekloge: Melibeus an des buochboums schat / Tityrum fry ligend funden hat / Der in vertriben tröstet wol / Lobt seine gaben als er sol. Ebd., fol. IIIv. $\mathrm{Zu}$ Reimübertragungen lateinischer Schultexte vgl. Nikolaus Henkel: Mittelalterliche Übersetzungen lateinischer Schultexte ins Deutsche. Beobachtungen zum Verhältnis von Formtyp und Leistung. In: Poesie und Gebrauchsliteratur im deutschen Mittelalter. Würzburger Colloquium 1978. Hg. von Volker Honemann u. a. Tübingen 1979, S. 164-179.

14 Worstbrock: Mulings Vergilübersetzung (Anm. 10), S. 209. 
tierung allgegenwärtig sind. Er transponiert das aus dem Bereich lateinischer Schullektüre geläufige Konzept der Texterschließung in die Volkssprache: Es handelt sich um „unterschiedliche schriftliche Ausprägungen des gleichen Verfahrens der Texterarbeitung “ ${ }^{15}$ Muling setzt nur die deutsche Sprache zur Förderung des Wortverständnisses und zum Erarbeiten des lateinischen Originals ein. Daher eignet sich seine Übersetzung wohl kaum zur Erschließung neuer Rezipientenkreise. Denn sie setzt die Benutzung des lateinischen Textes neben der deutschen Version voraus und bleibt insofern in der gleichen Umgebung beheimatet wie ihre Vorlage - im Bereich der schulischen Bildung: „Der Vorgang der Umsetzung in die Volkssprache dient also weitgehend nicht der Erschließung des Originaltextes für Benutzer, die diesen mangels entsprechender Sprachkompetenz nicht lesen können“. ${ }^{16}$

Aufschlussreich hinsichtlich der Wirkung seiner Übersetzung ist, was Muling am Schluss der Vorrede angibt (fol. IIr): Er habe die

hofnung sollichs nit verworfen noch veracht/ sonder als ander teutsche bücher gelesen. in guotem vffgenommen vnnd verstanden werde. So diß beschicht ouch andere seine [sc. Vergils] bücher mit höherm fleiß vnd ernstlicher vßlegung transferiern.

Wenn den deutschen Eklogen eine gute Aufnahme beim Zielpublikum vergönnt ist, dann würde Muling auch weitere Werke Vergils übersetzen und auf ähnliche Weise aufbereiten wollen. Ein Blick in die Rezeption von Mulings Übersetzungswerk der zehn Eklogen allerdings zeigt, dass es nie dazu gekommen ist: Weder existieren weitere Druckausgaben noch hat er andere Werke Vergils in derselben Form erschlossen. In dieser Hinsicht kann Mulings Bucolica-Übersetzung als durchaus erfolgloses Unternehmen gelten, das keine sichtbaren Spuren hinterlassen hat. ${ }^{17}$ Denn sein Verfahren ist im gesamten Mittelalter im Bereich der mündlichen Praxis, soweit wir sie aus den Handschriften rekonstruieren können, präsent und bedurfte wohl keiner so intensiven Überführung in die Schriftlichkeit.

15 Nikolaus Henkel: Deutsche Übersetzungen lateinischer Schultexte. Ihre Verbreitung und Funktion im Mittelalter und in der frühen Neuzeit. Mit einem Verzeichnis der Texte. München 1988 (Münchener Texte und Untersuchungen zur deutschen Literatur des Mittelalters 90), S. 118. 16 Ebd., S.2.

17 Mulings Übersetzung war wohl bis ins späte 18. Jahrhundert nicht mehr bekannt. Dafür spricht, dass Johann Friedrich Degen sie in seinem Kompendium der deutschen Übersetzungen lateinischer Klassiker nicht erwähnt, sondern Reichs Bucolica-Übersetzung als „älteste Verdeutschung der Eklogen“ anführt (Johann Friedrich Degen: Versuch einer vollständigen Literatur der deutschen Übersetzungen der Römer. Zweite Abteilung K-V. Altenburg 1797. Nachdruck Hildesheim u. a. 1997, S. 550). 
Im Jahr 1515 erschien bei Grüninger die erste deutsche Übersetzung von Vergils Aeneis, angefertigt vom Franziskanermönch Thomas Murner. ${ }^{18}$ Sie ist ebenso wie Mulings Werk mit den Holzschnitten aus Brants Vergilausgabe ausgestattet, die in Auswahl Murners Text begleiten. ${ }^{19}$

Eine bemerkenswerte Parallele zu Mulings Bucolica-Übersetzung ergibt sich bezüglich der in Murners Vorrede formulierten praktischen Nutzanwendung seiner Translation für die Arbeit mit dem Original. Murner intendiert die Lektüre des lateinischen Textes neben seiner Übersetzung: Mit Hilfe der deutschen Verse soll die Lektüre des lateinischen Textes on meister, ${ }^{20}$ also ohne Vermittlungsinstanz eines Lehrers bzw. des Lernzusammenhangs einer Schule ermöglicht werden. Dieses Verfahren zielt auf eine Vergleichbarkeit von Ausgangstext und Übersetzung, das in der Erstausgabe durch Marginalien unterstützt wird, die neben den deutschen Versen abschnittsweise die Initien der lateinischen Verse bzw. Halbverse der Aeneis bieten, auf die sich der deutsche Text jeweils bezieht. Murner betont explizit die am großen lateinischen Dichter erlernbare sprachliche Kompetenz: vnd was geweltigs dalmetschen sie dabei erlernen. ${ }^{21}$

Diese Aussagen implizieren eine wörtliche Übersetzungsweise, die den Nachvollzug der lateinischen Satzstruktur erlaubt und als Erschließungshilfe dient, wie sie Muling mit seiner Übersetzung bietet. Dem steht allerdings die Versform von Murners Translation entgegen: Denn die Entscheidung für deutsche Reimpaarverse fordert einen freieren, sinngemäßen Zugriff auf den Text. Dahinter steht das Bemühen, einen verständlichen deutschen Text zu schaffen, der dem lateinischen Original als eine - nach den Möglichkeiten der Zielsprache - formal gleichwertige Übersetzung an die Seite gestellt werden kann.

18 Murner (Anm. 2). Vgl. auch die aktuelle Untersuchung von Nikolaus Henkel: Vergil lesen. Thomas Murners Aeneis-Übersetzung als Weg zur Lektüre eines lateinischen Klassikers. In: Lehren, Lernen und Bilden. XXIII. Anglo-German Colloquium, Nottingham 2013 [im Druck].

19 Die Holzschnitte zur Aeneis sind in Werner Suerbaums Katalog der illustrierten Vergilausgaben knapp beschrieben, vgl. Werner Suerbaum: Handbuch der illustrierten Vergil-Ausgaben 1502-1840. Geschichte, Typologie, Zyklen und kommentierter Katalog der Holzschnitte und Kupferstiche zur Aeneis in Alten Drucken. Mit besonderer Berücksichtigung der Bestände der Bayerischen Staatsbibliothek München und ihrer Digitalisate von Bildern zu Werken des P. Vergilius Maro. Hildesheim, New York 2008 (Bibliographien zur klassischen Philologie 3), S.135-152.

20 Murner (Anm.2, fol. A1v) gibt an, er habe sein Werk vssenwendig mit verzeichnetem latyn versehen, das menglich schier on meister Vergilium lesen mög. Dass durch die Beigabe der lateinischen Initien eine bilinguale Lektüre intendiert ist, bemerkte schon Paul Scherrer: Thomas Murners Verhältnis zum Humanismus. Untersucht auf Grund seiner Reformatio Poetarum. Basel 1929, S. 25. Jüngst auch Henkel (Anm. 18).

21 Ebd., fol. A1v. 
Die deutsche Aeneis ist also in gewisser Weise eine hybride: Sie bietet zahlreiche Wortübersetzungen, die sich bis zur Unverständlichkeit eng an den lateinischen Text anschließen. Dabei wählt Murner aus dem sogenannten semantischen Fächer von möglichen je zu kontextualisierenden Bedeutungen eines Lexems eine Art von Grundbedeutung und gibt die eigentliche Erschließungsleistung dem Leser auf. ${ }^{22}$ Seine Intention besteht wohl darin, dem Leser tatsächlich die Möglichkeit eines Nachvollzugs der lateinischen Konstruktion zu bieten, wie er es in der Vorrede ankündigt. Andererseits zeichnet sich die Übersetzung durch eine freiere, auf einen kulturellen Transfer zielende Wiedergabe des lateinischen Textes aus. Durch die Versform und die zu einem großen Teil eigenständige, an der Umgangssprache orientierte Redeweise sowie durch das Bemühen um Verständlichkeit eröffnet Murners Übersetzung die lateinischen Bildungsinhalte erstmals einem am Wissen interessierten, nicht lateinisch gebildeten Publikum.

Während Muling mit seiner Übersetzung von Vergils Bucolica kaum Anklang gefunden hat, verhält es sich bei Murner anders: Nach Eckhart Bernstein habe er mit seiner Übersetzung „den Ton und den Geschmack der Zeit getroffen. “23 Für ihre Beliebtheit und intensivere Rezeption sprechen sieben Ausgaben bis $1606^{24}$ sowie die im Jahr 1610 postum publizierte Nachfolgeübersetzung des Augsburger Meistersingers Johannes Spreng, die sich an zahlreichen Stellen eng an Murners Text anlehnt. ${ }^{25}$

22 Die folgende kleine Auswahl an Beispielen mag die Praxis der Wortübersetzung veranschaulichen: incedunt pueri [...] ante ora parentum (Aen. 5,553; hier: ,Angesicht, vor den Augen'): Sie ranten vor ir vetter mund (5,1087); Parietibus [...] caecis (Aen. 8,589; hier: ,dunkle'): mit blinden wenden (5,1157); manus [...] Achaica (Aen. 5,623; hier: ,Schar'): Kriechisch handt (5,1224); Tectum [...] ingens (Aen. 7,170; hier: ,Haus'): ein grosses dach (7,329); captiui [...] currus (Aen. 6,184; hier: ,erbeutete'): gefanckne wegen (7,360). Die Verszählung bezieht sich auf die im Entstehen befindliche Edition von Murners Übersetzung, die synoptisch auch den Text der lateinischen Vorlage bieten wird. Sie wird in Freiburg im Rahmen eines DFG-Projekts unter der Leitung von Nikolaus Henkel von der Verfasserin vorbereitet. Zu Murners Übersetzungspraxis siehe auch Carola Redzich in diesem Band.

23 Bernstein (Anm. 5), S. 2.

24 Es sind die folgenden Ausgaben nachweisbar: Worms: Gregor Hofmann 1543 (VD16 V 1427); Worms: Gregor Hofmann [1543] (VD16 V 1428/1429); Frankfurt/M.: David Zöpfel 1559 (VD16 V 1430); Straßburg: Christian Müller d. Ä. 1559 (VD16 V 1431); Straßburg: Christian Müller d. Ä. [1559] (VD16 ZV 29820); Frankfurt/M.: David Zöpfel 1562 (VD16 V 1432); Jena, Leipzig: Johann Weidner für Johann Börner d. J. 1606 (VD17 1:043261R).

25 Im Kapitel zum Vergleich mit Johannes Sprengs Übersetzung sieht Bernstein die „starke Benutzung der Übersetzung Murners durch Spreng“ als „Beweis für die weite Verbreitung und den starken Einfluß der Übertragung des Franziskaners“, Bernstein (Anm. 5), S. 101. Zu Sprengs Aeneis-Übersetzung siehe auch Petra Fochler: Fiktion als Historie. Der Trojanische Krieg in der 


\section{Stephan Reichs Bucolica-Übersetzung}

Stephan Reich, oder auch Riccius, ${ }^{26}$ wie er sich nannte, stammte aus dem südlich von Jena gelegenen Kahla, wo er am 25. Dezember 1512 geboren wurde. Ab 1529 studierte er in Wittenberg Theologie, Philosophie und Mathematik, später auch das Recht. Zu seinen Lehrern gehörten Martin Luther, Justus Jonas, Caspar Cruciger und Philipp Melanchthon, den er besonders schätzte und mit dem ihn auch nach den Studienjahren eine enge Freundschaft verband. Er schloss seine Studien am 27. März 1536 mit dem Magistergrad ab und trat am Ende desselben Jahres in Jena sein erstes öffentliches Amt als Rektor der Stadtschule an. ${ }^{27}$ Einige Zeit später, im Jahr 1540, ging er nach Saalfeld in Thüringen, wo er ebenfalls als Rektor tätig war. Nach einigen Stellenwechseln und unglücklichen Zwischenfällen ${ }^{28}$ wurde er schließlich im Jahr 1559 Pfarrer der Propstei Lissen; er hatte dieses Amt bis zu seinem Tod inne.

Reich beherrschte nicht nur die lateinische, sondern auch die griechische Sprache. Im Jahr 1533 wurde er von Melanchthon an dessen Freund Johannes Stratius als Griechischlehrer vermittelt. Er versah diese Aufgabe zur großen Zufriedenheit beider Männer und kehrte im Jahr 1535 nach Wittenberg an die Universität zurück. ${ }^{29}$ Reichs Übersetzungstätigkeit nahm wohl in dieser Phase ihren

deutschen Literatur des 16. Jahrhunderts. Wiesbaden 1990 (Wissensliteratur im Mittelalter 4), S. 82-96.

$26 \mathrm{Zu}$ Leben und Werk Stephan Reichs vgl. Sven Limbeck: Reich (Riccius), Stephan. In: Frühe Neuzeit in Deutschland 1520-1620. Verfasserlexikon 5 (2016), Sp. 235-245; sowie die zwar in die Jahre gekommene, aber dennoch fundierte Gesamtwürdigung bei Ernst Koch: Über Mag. Stephan Reich (Riccius), sein Leben und seine Schriften. Meiningen 1886 (Einladungsschrift des Gymnasium Bernhardinum, Progr. Nr. 642), S. 5-40. Eine knappe Darstellung bietet Rudolf Herrmann: Luther als Mönch. Luthers Schüler in Thüringen (Konrad Limmer, Wolfgang Möstel, Stephan Reich, Bartholomäus Rosfeld, Lorenz Schmidt). Berlin 1952, S. 207-212.

27 Vgl. Herrmann (Anm. 26), S. 208.

28 Nach der Ernennung zum Diakon in Saalfeld (1542) wechselte Reich zunächst in die Pfarrei Langenschade (Ende 1545), danach als Diakon in seine Heimatstadt Kahla (1547). Dort suchte ihn großes Unglück heim: Im Jahr 1557 wurde seine Frau Barbara des Ehebruchs mit dem jungen Otto Gräfe beschuldigt; auch ihre gemeinsame Tochter soll involviert gewesen sein. Der Prozess zog sich bis Ostern 1558 hin: Gräfe wurde schließlich enthauptet, Frau und Tochter aus dem Herzogtum verwiesen. Reich hielt an der Unschuld seiner Frau fest und folgte ihr in die Verbannung, vgl. Koch (Anm. 26), S.19f. In den Jahrbüchern zur Geschichte der Stadt Kahla wird über den Prozess und seinen Ausgang berichtet, vgl. Jahrbücher zur Geschichte der Stadt Kahla. Hg. von Richard Denner. Kahla 1937, S. 88.

29 Vgl. Koch (Anm. 26), S. 6-8. 
Anfang: Er übertrug in den Jahren 1537 bis 1542 einige Reden des Demosthenes ins Deutsche, die er allerdings nicht zum Druck gab. ${ }^{30}$

Aus dem Bemühen, der studierenden Jugend das Verständnis der antiken Klassiker zu erleichtern, resultiert seine erste Aufbereitung von Vergils Bucolica für den Schulkontext: Die Quaestiunculae in Eclogas Virgiliii ${ }^{31}$ sind zugleich Reichs erstes Werk, das den Weg in den Druck fand. Es ist dem jungen Abraham Pfrundt gewidmet, dem Sohn des Amtmannes Egidius Pfrundt von Torgau, der ihm als Schüler zur wissenschaftlichen Ausbildung anvertraut war: Honesto adolescenti Abraham Pfrundt, Egidii praefecti Torgensis filio, discipulo suo carissimo (fol. a2r). Reichs Schrift sollte den jungen Schüler zu größerem Eifer anspornen (fol. a2r-v):

Sic enim tibi persuadeas velim, Abrahame cariss[ime] quod ista tuo nomini nuncupata sint, non tantum ut meum erga te, tuumque parentem, honestissimum virum, animum testarer, sed etiam ut te $[. .$.$] accenderem$

So möchte ich nämlich, dass du dich überzeugst, teuerster Abraham, dass diese [Quaestiones] deinem Namen gewidmet sind, nicht nur, damit ich meine gute Gesinnung dir und deinem Vater, dem ehrenvollsten Mann, gegenüber beweise, sondern auch damit ich dich ansporne. ${ }^{32}$

Nach Ausweis des Titels (Quaestiunculae [...] Iuventuti praescriptae) und der Vorrede entstammt die Schrift Reichs Unterrichtspraxis: Er soll ihren Inhalt seinen ehemaligen Schülern in Saalfeld zum Lernen diktiert haben (fol. a2r):

Cum in hac nostra schola Eclogas Virgilii honestis et mei et litterarum studiosis pueris praelegerem, aliquam studiorum meorum partem in id posui, ut in breves quaestiunculas locos communes, quos inprimis observari velim, conicerem.

Als ich in dieser unserer Schule die Eklogen Vergils den tugendhaften Schülern, die sowohl mir als auch den Studien eifrig ergeben sind, vorgelesen habe, habe ich einen Teil meiner Bemühungen darauf verwandt, in kurzen Fragen die loci communes beizufügen, die ich besonders beachtet haben wollte.

30 Es handelt sich um die drei Olynthischen Reden, das Schreiben des Königs Philipp an die Athener und Demosthenes' Antwort darauf sowie die vier Philippischen Reden, die Reich im Jahr 1537 aus dem Griechischen und teilweise aus einer lateinischen Übertragung Melanchthons ins Deutsche brachte. Aus den letzten Monaten des Jahres 1542 stammt Reichs Übersetzung der beiden Reden des Demosthenes gegen Aristogeiton. Die Handschriften befinden sich heute in der Forschungsbibliothek Gotha; vgl. Koch (Anm. 26), S. 9; Limbeck (Anm. 26), Sp. 238, 241.

31 Stephan Reich: Quaestiunculae In Eclogas Virgilii Salvendensi Iuventuti praescriptae. Frankfurt/M.: Peter Braubach 1546 (VD16 V 1490).

32 Die Übersetzungen lateinischer Zitate stammen hier wie auch sonst von der Verfasserin. 
Das Werk bietet daher einen interessanten Einblick in die Art der Vermittlung von Vergils Bucolica in der Schule, wie Reich sie handhabte: Es nütze nämlich sehr dem Verständnis, den Schülern schwierige Sachverhalte in Form von Fragen darzulegen: Prodest enim plurimum, res difficiles succinctis quaestionibus comprehensas pueris proponere (fol.a2r). $\mathrm{Zu}$ diesem Zweck bietet Reich zunächst lateinische Fragen und Antworten, die jede Ekloge hinsichtlich ihrer historischen Entstehungsumstände, der Terminologie, der Vorbilder, der Stilhöhe, der Personennamen, der Struktur u.a. besprechen, wobei das Hauptaugenmerk auf den loci communes liegt. ${ }^{33}$ Den Quaestiunculae folgen geschlossen die Argumenta Melanchthons, die den Inhalt jeder Ekloge auf ein bis zwei Seiten in lateinischer Prosa zusammenfassen (fol. d8v-e7v). Nach diesen gewissermaßen einführenden Partien wird schließlich der vergilische Originaltext geboten (fol. f1r).

Die Schrift wurde zwar nur einmal nachgedruckt, ${ }^{34}$ doch legte Reich ihren Inhalt seiner im Jahr 1565 erschienenen kommentierten Ausgabe von Vergils Bucolica zugrunde: Argumenta Seu Dispositiones Rhetoricae In Eclogas Virgilii. ${ }^{35}$ Sie basiert Kochs Ausführungen zufolge auf Vorlesungen Melanchthons, die Reich in seiner Wittenberger Studienzeit gehört hatte. ${ }^{36}$ Neben zwei Geleitgedichten des Lorbeerpoeten Johann Stigelius ${ }^{37}$ und der Widmungsvorrede an Herzog Johann Friedrich III. von Sachsen bietet die Ausgabe Melanchthons Argumenta zu den Eklogen, die aber nicht wie in den Quaestiunculae en bloc organisiert sind; vielmehr eröffnet den Vergiltext eine allgemeine Einführung Melanchthons: Quo consilio scripta sunt initio Bucolica P.M. (fol. B1v). Jeder Ekloge folgen sodann das jeweilige Argumentum und zahlreiche Auslegungen Melanchthons (dispositiones eiusdem, fol. B4r). Zur besseren Memorierbarkeit nimmt Reich die in je zwei elegischen Distichen gehaltenen Zusammenfassungen (Tetrasticha) der ersten bis

33 Folgendes Fragenspektrum ist zu verzeichnen: Quae res dedit occasionem Virgilio scribendi carmen Bucolicum? [...] Cur poeta inscripsit Bucolica? [...] Unde trahit originem? [...] Quem imitatus est poeta in Bucolicis? [...] Quid est Ecloga? [...] Quod genus dicendi in Eclogis secutus est? [...] Unde personae huius operis magna ex parte habent nomina? [...] Quot habet partes haec Ecloga? [...] Qui sunt in hac Ecloga loci communes digni observatione? Reich: Quaestiunculae 1546 (Anm. 31), fol. a3v-a7r.

34 Stephan Reich: Quaestiunculae In Eclogas Virgilii Salvendensi Iuventuti praescriptae. Frankfurt/M.: Peter Braubach 1548 (VD16 V 1494).

35 Stephan Reich: In Hoc Libello Continentur Argumenta Seu Dispositiones Rhetoricae In Eclogas Virgilii Autore Philip. Melanth. Item, Accesserunt Paraphrases, Ecphrases, et succinctae quaestiones in easdem Eclogas Autore M. Stephano Riccio. Weißenfels: Georg Hantzsch 1565 (VD16 V 1508).

36 Vgl. Koch (Anm. 26), S. 27.

37 Vgl. John L. Flood: Poets Laureate in the Holy Roman Empire. A Bio-Bibliographical Handbook. Bd. 4. Berlin, New York 2006, S. 2003-2007. 
achten Ekloge auf, die Stigelius in Wittenberg als Professor für Griechisch und Latein gelesen haben soll. ${ }^{38}$ Schließlich fügt er dem Werk eigene Prosa-Paraphrasen bei, die den Vergiltext zugleich mit einer Deutung versehen, sowie die Fragen und Antworten aus den Quaestiunculae, die nunmehr unter dem Titel Prolegomena In Bucolica Virgilii (fol. M3v) figurieren. ${ }^{39}$

An dieser intensiven Beschäftigung Reichs mit Vergils Bucolica lässt sich sein Bestreben ablesen, die studierende Jugend in das Verständnis des antiken Textes einzuführen. Die Übersetzung des Werkes - seine erste eines römischen Klassikers $^{40}$ - erscheint als nächster Schritt in diesem Vermittlungsprozess nur folgerichtig.

Reichs Übersetzung der Bucolica wurde - so die Angabe in der Widmungsvorrede - am 5. Februar 1567 fertiggestellt; ${ }^{41}$ der erste erhaltene Druck stammt aus dem Jahr $1568 .{ }^{42}$ Entsprechend der im Titel formulierten praktischen Nutzanwendung des Werkes (in usum puerorum) widmet Reich seine Übersetzung drei jungen Schülern, den Brüdern Johannes, Christoph und Valentin Stahls, den Söhnen des Dr. iur. utr. Johannes Stahls aus Halle an der Saale; ähnlich wie schon

38 Tetrasticha In Aliquot Eclogas Virgilii Memoriae causa, a D. Ioanne Stigelio conscripta, cum aliquando eas Vitebergae enarraret. Reich: Argumenta 1565 (Anm. 35), fol. G3r.

39 Das Werk wird abgeschlossen durch eine große Anzahl von Miszellaneen - kurze Abhandlungen verschiedenen Inhalts -, die Reich der Veröffentlichung für wert hielt: Miscellanea Stephani Riccii, In Quibus Variae historiae et fabulae dignae recitantur (fol. R1r). Die Schrift wurde im Jahr 1568 in einer erweiterten Fassung nochmals publiziert: Stephan Reich: In Hoc Libello Continentur Argumenta Seu Dispositiones Rhetoricae In Eclogas Virgilii Authore Philip. Melanch. Item, Accesserunt Paraphrases, Ecphrases, succinctae quaestiones et brevia Scholia Textus in easdem Eclogas Authore M. Stephano Riccio. Omnia recognita et aucta. Görlitz, Leipzig: Ambrosius Fritsch für Jakob Apel d. Ä. 1568 (VD16 ZV 25837). Ein weiterer Nachdruck erschien 1569 (VD16 V 1509).

40 Zuvor hatte er neben den Reden des Demosthenes Übersetzungen geistlicher Texte - vornehmlich Kirchenlieder und Psalmenauslegungen, aber auch eine Übersetzung von Luthers Auslegung des Propheten Micha - vorgelegt, vgl. Koch (Anm. 26), S. 12-22.

41 Aufgrund der Angabe in der Widmungsvorrede, jedoch ohne zwingende Argumente, nimmt Koch (Anm. 26), S. 32, einen ersten Druck für das Jahr 1567 an, der sich allerdings nicht nachweisen lässt. Ihm folgt Gronemeyer in seiner Bibliographie der deutschen Vergil-Übersetzungen, vgl. Horst Gronemeyer: Untersuchungen zur Geschichte der deutschen Vergil-Übertragung mit besonderer Berücksichtigung Rudolf Alexander Schröders. Hamburg 1963, S. 344. Im Kolophon des Druckes von 1573 heißt es: Finis Bucolicorum, quae primum germanice sunt reddita, et in publicum edita, Anno 1567. die 5. Februarii. Stephan Reich: Bucolica Virgilii In Usum Puerorum Germanice reddita per M. Stephanum Riccium. Leipzig: Jakob Bärwald (Erben) für Jakob Apel d. Ä. 1573 (VD16 V 1523), fol. R7v. Indes muss sich der Ausdruck in publicum edita nicht notwendig auf eine Druckversion beziehen.

42 Reich: Bucolica Virgilii (Anm. 3). 
die Quaestiunculae sollen die deutschen Eklogen den jungen Leuten als Ansporn dienen - Reich spricht von der adhortatio der optimi pueri (fol. A2v) und betont den großen Gewinn, den sein Werk für ihre Studien bereithalte: magnum fructum vestris studiis allaturus est (fol. C1v).

Eine Neuausgabe erschien bereits zwei Jahre später: ${ }^{43}$ Der Widmung an die Schüler geht nun eine Vorrede an den Leser (Ad Candidum Lectorem) voran; dadurch wird der in der Erstausgabe vorgestellte engere Kreis der primären Rezipienten zugunsten eines potentiell unfesten Publikums von Interessenten erweitert. In diesem Vorwort gibt Reich die Ursachen an, die ihn bewogen haben, Vergils Bucolica für die Jugend zu übersetzen (fol. A2r):

Cum Re Ipsa compererim, candide Lector, librum Bucolicorum Virgilii in omnibus scholis proponi pueris, et interdum a nonnullis praeceptoribus, praesertim indoctis, satis inepte reddi, putavi me aliquam gratiam initurum esse apud scholasticam iuventutem, si hunc libellum pro mea virili germanice redderem.

Als ich von dieser Sache erfahren habe, trefflicher Leser, dass das Buch der Bucolica Vergils in allen Schulen den Knaben vorgesetzt und bisweilen von einigen Lehrern, besonders den ungelehrten, unpassend genug vermittelt wird, glaubte ich, einige Gunst bei der studierenden Jugend zu erlangen, wenn ich dieses Büchlein nach meinem Vermögen deutsch wiedergeben würde.

Vergils Bucolica werden als ausgesprochene Schullektüre vorgestellt; nur wenige Lehrer seien jedoch in der Lage, sie angemessen zu vermitteln. Diesem Missstand soll Reichs Übersetzung abhelfen. Freilich verweist er auf einige Gegner, die seine Ansicht von der nutzbringenden Wirkung seiner Arbeit für das Verständnis des antiken Textes nicht teilen: Die Kritik beziehe sich jedoch nicht per se auf die volkssprachige Vermittlung der Bucolica, sondern vielmehr auf die Form der Übersetzung, die ungebundene Rede; durch die Umsetzung in deutsche Prosa ginge die Anmut der lateinischen Verse verloren. ${ }^{44}$

43 Stephan Reich: Bucolica Virgilii In Usum Puerorum Germanice Reddita. Per. M. Stephanum Riccium. Eisleben, Leipzig: Urban Gaubisch für Jakob Apel d. Ä. 1570 (VD16 V 1522).

44 Siehe ebd., fol. A2r: non defuerunt Zoili, qui consilium honestum et utile vituperarunt, eo quod humilitas argumenti Bucolici, sic insolutam orationem conversa gratiam amittat. (,Es fehlte nicht an Kritikern, die den ehrenvollen und nützlichen Entschluss deswegen getadelt haben, weil der schlichte Stil [das genus humile] des bukolischen Gedichts, wenn er in solch ungebundene Rede gebracht ist, die Anmut verliere.') 
Reich stellt die auf inhaltlicher wie sprachlicher Ebene unnachahmliche Vorbildhaftigkeit von Vergils Eklogen heraus, ${ }^{45}$ neben der seine Übersetzung als bescheidener Versuch fungiert, eine Hilfestellung zum Nachvollzug des lateinischen Textes zu bieten; ihr Wert liege daher in der pädagogisch-didaktischen Intention des Verfassers (fol. A2v):

Merebitur tamen laudem (ut optima quaeque mihi de te pollicear, candide Lector) conatus meus honestissimus, cum quicquid huius a me factum est, hoc consilio factum sit, ut puerorum studiis consulam.

Dennoch wird mein überaus ehrenvolles Unterfangen Lob verdienen (wie ich mir von dir, trefflicher Leser, gerade das beste erhoffe), weil alles, was in dieser Sache von mir getan wurde, dem Entschluss entstammte, den Studien der Knaben zu helfen.

Die Bezeichnung seines Unterfangens als res [...] novi exempli (fol. A2r) deutet darauf hin, dass Mulings Übersetzung zu dieser Zeit nicht mehr bekannt war. Dennoch ist es wenig erstaunlich, dass Reich sich im Grunde desselben translatorischen Verfahrens bedient, wie Muling es in seinem Hirten vnnd buren werck vorgelegt hatte; denn die Verwendung der Volkssprache als Hilfsmittel im didaktischen Vermittlungsprozess ist in Mittelalter und Früher Neuzeit neben der lateinischen Glossierung und Kommentierung Teil der Erschließungspraxis lateinischer Texte. Was Reich in schriftlich fixierter Form bietet, konnte in der Unterrichtssituation ad hoc vom Lehrer geleistet werden. Sein Übersetzungsverfahren soll im Folgenden am Beispiel der vierten Ekloge skizziert werden.

In Vergils vierter Ekloge, ${ }^{46}$ dem „Grundtext für die poetische Geschichtsdeutung der augusteischen Zeit“ ${ }^{47}$ bezieht der Dichter ein Sibyllenorakel auf seine Gegenwart und stellt Augustus in die typologische Nachfolge Saturns als Stifter der neuen aurea saecula. Der das Ende des eisernen Zeitalters und die Heraufkunft der gens aurea einläutende puer, der den Frieden bringen wird, erscheint als Präfiguration des Augustus:

45 Credo equidem, quod illa Maronis avena tenuis, suam quoque inimitabilem eruditionem, et artem habeat (ebd., fol. A2v). (,Ich glaube nämlich, dass jener zarte Gesang des Maro auch seine unnachahmliche Gelehrsamkeit und Kunst hat.')

46 Vgl. hierzu die grundlegende Textuntersuchung von Walther Kraus: Vergils vierte Ekloge: Ein kritisches Hypomnema. In: Aufstieg und Niedergang der römischen Welt II 31,1: Principat. Sprache und Literatur. Hg. von Wolfgang Haase. Berlin 1980, S. 604-645.

47 Ulrich Schmitzer: Friede auf Erden? Latinistische Erwägungen zur pax Augusta in interdisziplinärer Perspektive. Antrittsvorlesung 2004, S. 5, http://edoc.hu-berlin.de/humboldt-vl/148/all/ PDF/148.pdf (6.3.2015). 
Sicelides Musae, paulo maiora canamus!

non omnis arbusta iuvant humilesque myricae;

si canimus silvas, silvae sint consule dignae.

Ultima Cumaei venit iam carminis aetas;

magnus ab integro saeclorum nascitur ordo.

iam redit et Virgo, redeunt Saturnia regna,

iam nova progenies caelo dimittitur alto.

tu modo nascenti puero, quo ferrea primum

desinet ac toto surget gens aurea mundo,

casta fave Lucina, tuus iam regnat Apollo. (Ecl. 4,1-10 $)^{48}$

Sizilische Musen, lasst uns ein wenig Erhabeneres besingen! Nicht alle erfreuen Büsche und niedrige Tamarisken. Wenn wir Wälder besingen, sollen die Wälder eines Konsuls würdig sein. Schon ist die letzte Zeit des cumaeischen Liedes gekommen; die große Reihe der Zeitalter entsteht von neuem. Schon kehrt die Jungfrau zurück, die Herrschaft des Saturn kehrt wieder, schon wird ein neues Geschlecht vom hohen Himmel gesandt. Du, reine Lucina, sei gnädig dem Knaben, der soeben geboren wird, mit dem das eiserne Geschlecht zuerst vergehen und das goldene sich in der ganzen Welt erheben wird; schon herrscht dein Apollon.

\section{Bei Reich lautet die Stelle (fol. E8v-F1v):}

Sicelides Musae, O jr lieben Muse/ canamus, wir wöllen ein mal schreiben/ paulo maiora, von tapffern sachen/ das ist/ Wir wollen ein wenig ein hoher Liedlein singen/ non omnes, nicht jederman/ iuvant, hat lust vnd gefallen/ oder höret gerne/ Arbusta et humiles myricae, von hecken vnd Tamarisken stauden/ oder/ von Ziegen vnd Böcken/ si canamus silvas, wenn ich etwas höhers schreibe/ oder/ so ich von Königs Sönen singe/ Silvae sint consule dignae, So sol man auch in solchem Gesang oder schreiben einen Burgermeister loben vnd preisen.

Iam venit, Jtzt kömpt oder ist verhanden/ ultima aetas, die letzte zeit/ oder das letzte alter/ carminis Cumaei, nach der prophecey Sybille/ oder/ von welcher Sybilla propheceiet hat/ Magnus ordo, eine grosse verenderung/ seculorum, der Welt/nascitur, wil anfahen/ ab integro, auff ein newes/ oder/ von forn an/ das ist/ nu wird gar wider eine newe Welt geschaffen/ iam redit, Jtzt kömpt auch wider/ virgo. s. iusticia, die Gerechtigkeit/ redeunt, Es kömpt auch wider/ saturnia regna, das reiche Regiment/ das vnter dem Saturno gewesen ist/ iam dimittitur, Jtzt kömpt/ oder wird gesand/ alto caelo, vom hohen Himmel/ nova progenies, ein newe Geburt/ oder/ ein newgeboren Geschlecht.

Tu modo casta Lucina, Allein du o keusche oder heilige Lucina vnd Wehefraw/ fave, sey gnedig/ oder/ gib glück/ nascenti puero, dem newgebornen Kindlein/ quo, zu welches Kindlein zeit/ ferrea gens, die böse Leute/ oder/ die arge Welt/ desinet, wird erstlich auffhören/ Ac, darnach/ gens aurea, die frommen Leute/ oder/ die güldene Zeit/ surget, werden sich erheben/ oder/ wird wasschen [sic] vnd entstehen/ toto mundo, so weit die gantze Welt ist/ oder/ in der gantzen Welt/ Tuus iam regnat Apollo, Jtzt regieret der rechte Man/ oder/ jtzt sitzet im reich dein bruder Augustus.

48 P. Vergili Maronis Opera. Hg. von Roger A. B. Mynors. Oxford 1969 (Oxford Classical Texts). 
Reichs Übersetzung ist mit dem lateinischen Originaltext so verschränkt, dass den kurzen, nach der syntaktischen Abhängigkeit, dem ordo naturalis, organisierten lateinischen Syntagmen jeweils Wort für Wort eine deutsche Entsprechung folgt. Teils orientiert sich die Übersetzung an der Zielsprache und bietet insofern interpretierende Elemente: Sicelides Musae, O jr lieben Muse/ canamus, wir wöllen ein mal schreiben/ paulo maiora, von tapffern sachen; teils wiederum folgt sie wörtlich dem lateinischen Text: alto caelo, vom hohen Himmel/ nova progenies, ein newe Geburt. An vielen Stellen werden verschiedene Übersetzungsmöglichkeiten geboten, die ein oder kennzeichnet: ultima aetas, die letzte zeit/ oder das letzte alter. Wenn man jeweils die eine oder andere Alternative liest, ist die deutsche Übersetzung syntaktisch kohärent: gens aurea, die frommen Leute/ oder/ die güldene Zeit/ surget, werden sich erheben/ oder/ wird wasschen [sic] vnd entstehen. Auf die integrierte Ebene der Auslegung und Kommentierung des Vergiltextes wird mittels das ist-Formeln verwiesen: paulo maiora, von tapffern sachen/ das ist/ Wir wollen ein wenig ein hoher Liedlein singen.

Reichs Übersetzung ist dem lateinischen Ausgangstext deutlich untergeordnet; angestrebt ist der präzise Nachvollzug des Originals im Medium der Volkssprache. Durch die Umformung des lateinischen Textes in den ordo naturalis und seine syntaktische Erschließung durch eine bewusst wörtliche und kleinschrittig verfahrende Translation wird die Hinführung zum Verständnis des vergilischen Textes erleichtert. ${ }^{49}$ Dieser wird zwar geboten, doch so segmentiert, dass von der Anmut der lateinischen Verse tatsächlich nichts bleibt. Insofern setzt diese Übersetzung die Benutzung des Originaltextes neben der deutschen Version voraus. Es ist denkbar, dass Reich sie für den Schulunterricht als ergänzendes Hilfsmittel zu seiner kommentierten Bucolica-Ausgabe konzipierte, ${ }^{50}$ zumal die Übersetzung nur wenige Jahre nach dieser Ausgabe entstand. Für den Erfolg dieses dualen Vermittlungsprozesses spricht, dass Reich die im Jahr 1573 erschienene Ausgabe der Bucolica-Übersetzung ${ }^{51}$ um eine umfangreiche Einführung, die unter anderem eine deutsche Fassung der Quaestiunculae bietet, erweitert hat: Ein kurtzer vnter-

49 Dieses Verfahren lässt sich bis ins frühe Mittelalter nachweisen: Notker III. von St. Gallen ordnet den Text von Boethius' Consolatio Philosophiae nach der „syntaktischen Dependenz“ und übersetzt ihn „Phrase für Phrase in die Muttersprache der Schüler“. Nikolaus Henkel: Übersetzen im Mittelalter. In: Geschichte der Übersetzung. Beiträge zur Geschichte der neuzeitlichen, mittelalterlichen und antiken Übersetzung. Hg. von Bogdan Kovtyk, Hans-Joachim Solms, Gerhard Meiser. Berlin 2002 (Angewandte Sprach- und Übersetzungswissenschaft 3), S. 191-214, hier S. $199 \mathrm{f}$.

50 Reich: Argumenta 1565 (Anm. 35).

51 Reich: Bucolica Virgilii (Anm. 42). 
richt der Jugendt zum besten gestellet/ warumb der Poet Virgilius sein Buch/ das er nennet Bucolica/ geschrieben habe/vnd was der Inhalt darinne sey (fol. C2v).

Den deutschen Bucolica war einiger Erfolg beschieden: Bis 1612 lassen sich zehn weitere Drucke nachweisen, die trotz der lokal auf Leipzig, Eisleben und Erfurt beschränkten Verbreitung auf eine durchaus rege Nachfrage schließen lassen. ${ }^{52}$

\section{Stephan Reichs Georgica-Übersetzung}

Angesichts dieses Erfolgs seiner Übersetzung bereitete Reich auch Vergils Georgica auf dieselbe Weise für den Schulgebrauch auf. Ihre deutsche Fassung erschien nur kurze Zeit nach der Translation der Bucolica in zwei Teilen zu je zwei Büchern. ${ }^{53}$ Im Titel wird der gleiche Nützlichkeitsanspruch formuliert: in usum studiosae iuventutis. Reich widmet die im Jahr 1571 erschienene Übersetzung der ersten beiden Georgica-Bücher drei jungen Schülern, den Brüdern Ernfried, Heinrich und Gottfried von Ende. ${ }^{54}$ Die umfangreiche Vorrede liefert interessante Einblicke in die Gelehrtenkultur der Zeit: Hatte Reich im Vorwort seiner deutschen Bucolica auf die Kritiker (zoili) aufmerksam gemacht, denen die sprachliche Form der Umsetzung ein Dorn im Auge sei, so führt er diesen Sachverhalt nun weiter aus. Einerseits treffe der Tadel ihn als Person, da er sich von seinen geistlichen Aufgaben - zu dieser Zeit war er Pfarrer in Lissen - durch seine Arbeiten für die Schule ablenken lasse. Andererseits sind es gerade diese Werke, deren Stil insbesondere den Gelehrten allzu dürftig und kindisch erscheine - kurz: eines Vergil nicht würdig; ${ }^{55}$ ein Umstand, auf den Reich in seiner Bucolica-Übersetzung bereits hingewiesen hatte. ${ }^{56}$ Seine folgende ausführliche Rechtfertigung spiegelt

52 Vgl. die nachgewiesenen Ausgaben bei Limbeck (Anm. 26), Sp. 242.

53 Reich: Virgilii priores duo libri Georgicorum (Anm. 3); Reich: Virgilii posteriores duo libri Georgicorum (Anm. 3).

54 Nobilitate Generis Et Praeclara Ingenii Indole ornatissimis adolescentibus Ernfrido, Henrico et Gotfrido ab Ende fratribus, nobilitate ac omni virtutum genere praestantissimi viri Gotfridi ab Ende in Vuildeborn et Lobichaw filiis. Reich: Virgilii priores duo libri Georgicorum (Anm. 3), fol. A2r.

55 Alii non decere meam personam aiunt, cum ministerio Ecclesiastico fungar, ut animum Scholasticis istis operis occupatum habeam. Nonnulli labores meos, quos in exponendis veterum autorum scriptis praestare soleo, nimis tenues et pueriles esse calumniantur (ebd., fol. A2v). (,Die einen sagen, dass es sich für meine Person nicht zieme, dass ich mich, während ich ein kirchliches Amt ausübe, mit diesen Schulschriften befasse. Einige kritisieren meine Arbeiten, die ich zur Erklärung der Schriften antiker Autoren vorzulegen pflege, als allzu dürftig und kindisch.`)

56 Dort schreibt er an die Widmungsträger: Transmitto vobis etiam Bucolica [...] germanice a me [...] reddita, qui labor etsi puerilis videatur, tamen magnum fructum vestris studiis allaturus est. 
die Intensität der Kontroverse: Unter Berufung auf biblische Autoritäten verweist Reich auf die seit jeher bestehende Koppelung von Schule und Kirche; Letzterer würden diejenigen einmal besser dienen, die eine fundierte Bildung erhielten. ${ }^{57}$ Diese könne aber auf keine andere Weise besser gewährleistet werden als durch das Studium antiker Autoren: Quod cum nulla ratione rectius fieri possit, quam si apud adolescentes studiosos veterum autorum lectio conservetur (fol. A3v). Daher habe Reich seine gesamte freie Zeit, die ihm der Beruf und die damit verbundenen Aufgaben ließen, darauf verwandt, den Schülern die vorbildlichen Klassiker zu erklären: si quid mihi temporis a vocationis meae laboribus propriis, superest, id ad exponendos atque illustrandos bonos scriptores [...] confero (fol. A4v-A5r).

Seines schlichten Stils ist der Übersetzer sich zwar durchaus bewusst, verweist jedoch auf dessen Funktionalität für den intendierten Adressatenkreis des Werkes: Es richte sich nicht an die gelehrten Männer (viri doctissimi), sondern sei hauptsächlich zum Nutzen der studierenden Jugend und als Hilfsmittel für die noch bescheidenen Fähigkeiten der Lernenden verfasst: ad iuventutis scholasticae utilitatem et ad iuvanda tenera ingenia discentium potissimum a me scripta (fol. A5v). Der Lernerfolg hänge nämlich maßgeblich von einer dem Wissensstand der Schüler angemessenen Behandlung des Stoffes ab; der Unterricht sei nur dann fruchtbringend, wenn der Lehrer sich dem Auffassungsvermögen und den geistigen Anlagen seiner Schützlinge anpasse: Non igitur feliciter pueros docebit nisi qui ad captum, et tenera ipsorum ingenia se accomodaverit (fol. A5vA6r). Durch die adäquate Unterweisung der Schüler in seinen Schriften diene Reich der kirchlichen Institution - so die Summa seiner Rechtfertigung.

Der zweite Teil der Georgica-Übersetzung ist als Neujahrsgabe ${ }^{58}$ den Schülern Michael, dem Sohn des Zeitzer Bürgers Peter Lemmermann, und Christopher, dem Sohn des Bartholomäus Georg aus Dreißig, gewidmet. ${ }^{59}$ Nach Auskunft der

Reich: Bucolica Virgilii (Anm.3), fol. C1v. (,Ich übersende euch auch die Bucolica von mir auf Deutsch wiedergegeben; auch wenn diese Arbeit kindisch erscheinen mag, wird sie dennoch euren Studien einen großen Gewinn bringen.')

57 Primum constat semper Eccelsiae Scholas coniunctas fuisse, ut historiae Prophetarum, Christi et Apostolorum ostendunt [...] Ita in scholis ingenia educari necesse est, quae Ecclesiae aliquando salutariter serviant. Reich: Virgilii priores duo libri Georgicorum (Anm. 3), fol. A3r-v. (,Zunächst steht fest, dass Schulen immer der Kirche verbunden waren, wie die Geschichten der Propheten, Christi und der Apostel zeigen. Es ist nötig, begabte Köpfe in den Schulen so auszubilden, dass sie irgendeinmal der Kirche auf heilsame Weise dienen.')

58 So die Auskunft am Ende der Vorrede: Datae ex praepositura Lyssena die natali Christi, inchoante annum 1572. Reich: Virgilii posteriores duo libri Georgicorum (Anm. 3), fol. A3v.

59 Siehe ebd., fol.)(2r: Eximiae Spei Praeclaraeque Indolis Adolescentibus Michaeli Petri Lemmermanni civis Zizensis filio et Christophoro, Bartholomei Georgii Quaestoris in Draissig filio. 
Vorrede, in der eine ausführliche Auslegung des Bienenstaates aus dem vierten Buch der Georgica im Zentrum steht, soll das Werk den jungen Leuten sowohl Unterhaltung als auch Nutzen bringen, sie aber auch bei ihren Studien anspornen (fol. A3r):

\footnotetext{
Proinde placuit et posteriores duos libros Georgicorum vernacula lingua interpretari eosque vestro nomini, charissimi adolescentes, nuncupare, partim quod consideratio ipsa apicularum plurimum habeat delectationis et fructus, partim ut vos in urgendis studiis exemplo apum ad sedulitatem incitem.
}

Daher habe ich beschlossen, auch die nachfolgenden zwei Bücher der Georgica in die Volkssprache zu übersetzen und sie eurem Namen, teuerste Jünglinge, zu widmen, teils, weil die Betrachtung der Bienen selbst sehr viel Genuss und Nutzen birgt, teils, damit ich euch nach dem Beispiel der Bienen zu unermüdlichem Fleiß, die Studien eifrig zu betreiben, ansporne.

Die Übersetzungspraxis der Georgica ist den Bucolica vollständig vergleichbar: Die mit dem lateinischen Text verschränkte deutsche Übersetzung bietet eine entsprechend wortgenaue Wiedergabe der kurzen Kola und ist um alternative Übersetzungsmöglichkeiten sowie die Ebene der Kommentierung angereichert. Zwar erschienen zwischen 1580 und 1627 in Leipzig drei Neuauflagen, ${ }^{60}$ dennoch steht die Georgica-Übersetzung hinsichtlich ihrer Verbreitung und Wirkung hinter Reichs Translation der Eklogen zurück. Das mag freilich damit zusammenhängen, dass die Bucolica in dieser Zeit als Schullektüre weiter verbreitet waren, wie Reich dies in der Vorrede zu ihrer Übersetzung angibt.

\section{Zur Vergil-Lektüre im 16. Jahrhundert}

Von der Erstausgabe (Rom 1469) bis 1500 sind 172 Vergilausgaben nachweisbar; ${ }^{61}$ im 16. Jahrhundert dürften es kaum weniger sein. Die vergleichsweise geringe Zahl an Ausgaben deutscher Übersetzungen, die dieser Masse gegenüberstehen, legt nahe, dass Vergil im 15. und 16. Jahrhundert noch weitgehend in lateinischer Sprache gelesen wurde. Deshalb hat Mulings Übersetzung wohl keine Verbreitung gefunden: Seine wörtliche translatorische Praxis, begleitet von einer ProsaParaphrase und kommentierenden Elementen, entspricht den Verfahren der

60 Vgl. Limbeck (Anm. 26), Sp. $242 \mathrm{f}$.

61 Die Zahl bezieht sich auf Opera-, Aeneis-, Bucolica- und Georgica-Ausgaben, vgl. Martin Davies, John Goldfinch (Hg.): Vergil. A Census of Printed Editions 1469-1500. London 1992. 
Texterschließung in dieser Zeit, wie sie in den Lateinschulen praktiziert wurde. Was dort in lateinischer Sprache erfolgt, transponiert Muling lediglich ins Deutsche. Die mangelnde Verbreitung seiner Übersetzung zeigt, dass daran kein Bedarf bestand.

Reich bedient sich bei seiner Bucolica-Übersetzung grundsätzlich des gleichen Verfahrens wie Muling, das im Schulkontext ubiquitär war: Der Vergiltext wird kleinschrittig durch eine wortgetreue Translation und die Beigabe kommentierender Elemente erschlossen. Allerdings hatte Reich mit seiner Übersetzung einigen Erfolg, den die später ins Deutsche gebrachten Georgica bestätigen. Auch wenn ihre Vorrede dokumentiert, dass Reichs Verfahren wohl nicht unumstritten war, so lässt sich doch an der Verbreitung der Bucolica-Übersetzung in zahlreichen Ausgaben ein Wandel der Lektürepraktiken ablesen: Erfolgte die Vergillektüre in der Schule zu Beginn des 16. Jahrhunderts noch überwiegend lateinisch, so gewinnt in dessen zweiter Hälfte - nicht zuletzt unter dem Einfluss der Reformation - die deutsche Sprache als Medium der Vermittlung zunehmend an Bedeutung.

Murners Übersetzung von Vergils Aeneis scheint zwar auch erst nach der Reformation größere Verbreitung gefunden $\mathrm{zu}$ haben, ${ }^{62}$ doch ist sie nicht primär im Bereich der schulischen Bildung zu verorten, sondern richtet sich durch die Versform und die stellenweise freiere Wiedergabe an einen potentiell erweiterten Rezipientenkreis. In dieser Hinsicht ist sie nicht mit den ausgesprochenen,Schultexten' Mulings und Reichs vergleichbar. Anhand ihrer Bucolica-Übersetzungen jedoch lassen sich durchaus Veränderungen im Hinblick auf die Art und Weise der Vergillektüre in der Schule des 16. Jahrhunderts verfolgen.

62 Der erste Nachdruck erschien fast 30 Jahre nach dem Erstdruck in Worms, wobei die Widmungsvorrede sowie der Name des Franziskaners - ein erbitterter Gegner Luthers - fehlen, was wohl dem Einfluss der Reformation zuzuschreiben ist. Vier weitere Ausgaben stammen aus der zweiten Hälfte des 16., eine aus dem Beginn des 17. Jahrhunderts, vgl. oben, Anm. 24. 